\title{
Retrospective Study to Investigate the Effect of Fixed Ratio Management of Packed Red Blood Cell to Fresh Frozen Plasma on Transfusion Requirements in Postpartum Hemorrhage
}

Carolyn Weiniger ${ }^{1}$, Noa Yakirevich ${ }^{1}$, Alexander Gural ${ }^{1}$, Hen Sela ${ }^{2}$, Sharon Einav ${ }^{2}$

${ }^{1}$ Hadassah University Medical Center, Jerusalem,

${ }^{2}$ Shaare Zedek Medical Center, Jerusalem

Israel

Background: Postpartum hemorrhage (PPH)
is a major cause of maternal morbidity, and
often necessitates blood product
transfusion. Outcome and survival is
improved among trauma victims using a
transfusion protocol of $1: 1$ fixed ratio for
packed red blood cells (PRBC) to fresh
frozen plasma (FFP), and $1: 1$ fixed ratio has
been recommended for PPH despite poor
evidence.(1) Our primary aim was to study
transfusion requirements among women
with PPH managed with a $1: 1$ fixed ratio
PRBC:FFP versus nonfixed ratio.
Methods: Retrospective IRB approved study in 2 neighboring tertiary centers that cover $80 \%$ of labors in the metropolitan area. We identified PPH cases, $\geq 3$ PRBC within $24 \mathrm{hr}$ of delivery, from blood bank records. We focused on the active bleeding period (blood products transfused continuously until a $4 \mathrm{hr}$ break without blood product administration was identified). Massive transfusion protocol (MTP) affirming 1:1 fixed ratio PRBC:FFP was introduced in 2010 in both centers. Demographic, obstetric, and blood management data were retrieved.

We compared estimated blood loss (EBL), blood product administration, and hematologic variables for PPH managed by 1:1 fixed ratio PRBC:FFP versus nonfixed ratio, using descriptive statistics.

Results: We identified 273 women (20042014) with PPH $\geq 3$ PRBC within 24hr of delivery. 1:1 fixed ratio PRBC:FFP management was used for $41 / 161$ (26\%) women since 2010 vs. $23 / 112$ (21\%) prior to 2010 , p=0.34. EBL among women managed with $1: 1$ fixed ratio was $2.3 \pm 0.7 \mathrm{~L}$ vs. $2.9 \pm 1.9 \mathrm{~L}$ with nonfixed ratio, $\mathrm{p}=0.04$. PPH managed with $1: 1$ fixed ratio received less PRBC units, median(IQR) 5(46) vs. 6(410) with nonfixed ratio, $p=0.013$, Table 1. Nadir fibrinogen levels for $1: 1$ fixed ratio were significantly higher, $324.0 \pm 131.3 \mathrm{mg} / \mathrm{dl}$ vs. $264.6 \pm 113.5 \mathrm{mg} / \mathrm{dl}$ with nonfixed ratio, $\mathrm{p}=0.003$, despite similar baseline values, Table 2. PPH management with nonfixed ratio PRBC:FFP was associated with a higher likelihood of receiving massive transfusion ( $\geq 8 \mathrm{PC}$ units), Odds Ratio $2.8895 \% \mathrm{Cl} 1.376 .06$.

Table 1: Blood transfusion management for 1:1 fixed ratio PRBC:FFP versus nonfixed ratio among women with postpartum hemorrhage

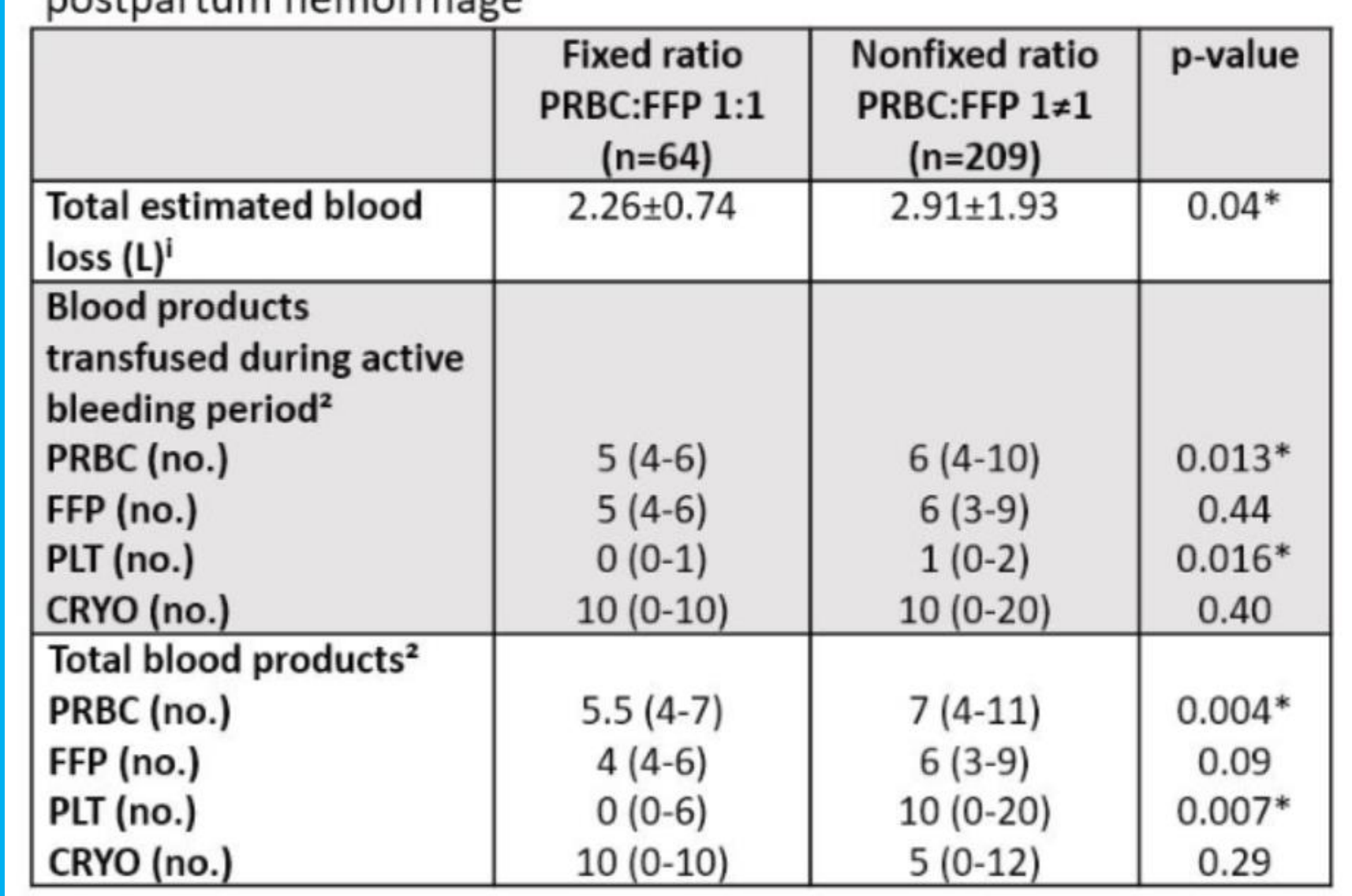

Key: PRBC=Packed red blood cells, FFP=Fresh frozen plasma, $\mathrm{CRYO}=$ Cryoprecipitate, $\mathrm{PLT}=$ Platelets; no.=number of units; 'Mean \pm SD, ${ }^{2}$ Median (IQR); ${ }^{*}=\mathrm{p}<0.05$, significant difference
Table 2: Hematologic values for 1:1 fixed ratio PRBC:FFP versus nonfixed ratio among women with postpartum hemorrhage

\begin{tabular}{|c|c|c|c|}
\hline & $\begin{array}{l}\text { Fixed ratio } \\
\text { PRBC:FFP 1:1 } \\
(n=64)\end{array}$ & $\begin{array}{c}\text { Nonfixed ratio } \\
\text { PRBC:FFP } 1 \neq 1 \\
(n=209)\end{array}$ & p-value \\
\hline Fibrinogen $(\mathrm{mg} / \mathrm{dl})^{i}$ & & & \\
\hline Baseline & $498.5 \pm 180.7$ & $481.5 \pm 154.7$ & 0.75 \\
\hline $\begin{array}{l}\text { First during active } \\
\text { bleeding period }\end{array}$ & $360.1 \pm 134.6$ & $311.7 \pm 131.2$ & 0.050 \\
\hline Nadir & $324.0 \pm 131.3$ & $264.6 \pm 113.5$ & $0.003^{*}$ \\
\hline Hemoglobin $(g / d l)^{i}$ & $115+14$ & $116+14$ & 0.59 \\
\hline $\begin{array}{l}\text { First during active } \\
\text { bleeding period }\end{array}$ & $9.1 \pm 1.8$ & $9.2 \pm 1.7$ & 0.67 \\
\hline Nadir & $7.8 \pm 1.5$ & $7.4 \pm 1.5$ & 0.07 \\
\hline Hematocrit (\%) & & & \\
\hline Baseline & $34.1 \pm 3.9$ & $34.7 \pm 3.8$ & 0.32 \\
\hline $\begin{array}{l}\text { First during active } \\
\text { bleeding period }\end{array}$ & $27.1 \pm 5.3$ & $27.2 \pm 5.1$ & 0.84 \\
\hline Nadir & $23.1 \pm 4.6$ & $21.9 \pm 4.3$ & 0.06 \\
\hline
\end{tabular}

Key: PRBC=Packed red blood cells, FFP=Fresh frozen plasma; 'Mean \pm SD; ${ }^{*}=p<0.05$, significant difference

Conclusion: Introduction of the MTP did not increase use of $1: 1$ fixed ratio PRBC:FFP for PPH management. Management of PPH using a 1:1 fixed ratio PRBC:FFP strategy was associated with significantly lower EBL and less PRBC and platelets, lower likelihood of massive transfusion and higher nadir fibrinogen levels. Our study findings support using 1:1 fixed ratio PRBC:FFP administration for PPH management. $(1,2)$ 